\title{
Photoplastic effect in polycarbonate using microhardness measurements
}

\author{
R BAJPAI*, SANDHYA SHARMA, V K VASTAL and B P CHANDRA \\ Department of Postgraduate Studies and Research in Physics, Rani Durgavati University, Jabalpur 482 001, India
}

MS received 6 August 2002; revised 5 April 2003

\begin{abstract}
Vickers microhardness indentation technique has been employed to detect the photoplastic effect in the transparent polycarbonate specimens in darkness and under mercury illumination. For low applied loads, the hardening of specimens under illumination confirms the positive photoplastic effect that causes illumination-induced increase of crosslinking (in polymer). For high applied loads the positive photoplastic effect decreases as the level of microhardness decreases.
\end{abstract}

Keywords. Photoplastic effect; polycarbonate; microhardness.

\section{Introduction}

Photoplasticity or the so-called nonlinear photomechanics can in general be defined as a full-field technique that is based on the interpretation of the optical patterns in transparent birefringent materials that are or were stressed beyond their elastic limit. Nowadays researchers are very much interested in the nonlinear character of materials. The photoplastic method using transparent polymers is a very reliable method to simulate the elastoplastic behaviour of prototype materials.

Ito (1962) was the first to suggest the use of polycarbonate for photoplastic models. Brill (1966) then used the same material to perform basic studies in one and two-dimensional photoplasticity. He found that the isochromatic parameter is a function of the principal strain difference and that the isoclinic parameters provide information about the principal strain directions.

Polymers are an important engineering material in single-phase application as well as the matrix in composites. Plastic deformation plays a key role in various instability phenomena such as shear bonding, necking, crazing, etc which influence the failure mechanism of polymers. The optical and mechanical behaviour of polymers depend basically on the their viscous character.

Such an effect, which may be called photomechanical effect, was indeed found by studying the microhardness of polycarbonate under dark and illumination. Polycarbonate is a hetero chain thermoplastic possessing high impact strength, good heat resistance and self-extinguishing properties. Mills (1976) and Zurimendi et al (1982) have studied some mechanical properties of polycarbonate such as impact and tensile strengths. Observations have

*Author for correspondence been made regarding its hardness (Bajpai and Datt 1986). However, no observations have been made regarding the influence of illumination on the microhardness of polycarbonate to study the photoplastic effect in this material.

Generally, as for other mechanical properties, microhardness undergoes both transient and permanent changes as a result of absorption of energy in the form of radiation (Lopez 1993). Microhardness seems to be especially suited in monitoring the surface phenomenon of radiation induced effect on polymers because it investigates the surface of the materials and is a non-destructive technique for studying the various physical properties of polymers (Gonzalez et al 1986).

\section{Experimental}

Polycarbonate specimens, $1 \mathrm{sq} . \mathrm{cm}$ in size, cut from transparent polycarbonate sheet of thickness $3 \mathrm{~mm}$ were utilized in the present investigation supplied by $\mathrm{M} / \mathrm{s}$ Chemical Agencies, Mumbai. The indentations were carried out by mhp 160 microhardness tester with a Vickers diamond pyramidal indenter attached to Carl Zeiss NU2 microscope. A 100 watt condensed mercury lamp was used as the source of light. The specimen was fixed in such a way that the surface to be indented was perfectly horizontal. The applied load was varied from 10 to $100 \mathrm{~g}$. It was applied very slowly and at a steady rate. The time of indentation was kept at $30 \mathrm{~s}$ in each case. The diameter of the indentations were then measured using a micrometer eyepiece with an objective of magnification $\times 25$. The illuminating lamp was placed at a desired distance from the specimen for fixed exposure time. It should be added that the light source was never in contact with any part of the hardness tester during the experiment. Vickers hardness number, $H_{\mathrm{v}}$, is measured by using the relation 


$$
H_{\mathrm{v}}=1 \cdot 854 \times \frac{L}{d^{2}}\left(\mathrm{~kg} / \mathrm{mm}^{2}\right)
$$

where $L$ is load in $\mathrm{kg}$ and $d$ the diameter of indentation in $\mathrm{mm}$. For the same load at least 5 indentations were made and average $H_{\mathrm{v}}$ was computed.

\section{Results and discussion}

\subsection{Microhardness of polycarbonate without illuminations}

Figure 1 illustrates (without illumination) the variation of $H_{\mathrm{v}}$ in polycarbonate at various loads. It reveals that initially, $H_{\mathrm{v}}$, gradually increases with increasing load and tends to reach a saturation value of $8.47 \mathrm{~kg} / \mathrm{mm}^{2}$ at $30 \mathrm{~g}$. It again starts increasing at $40 \mathrm{~g}$ and acquires the saturation value of $9.02 \mathrm{~kg} / \mathrm{mm}^{2}$ at $60 \mathrm{~g}$. Finally, the microhardness tends to become almost independent of load as no change in $H_{\mathrm{v}}$ was observed above the load of $80 \mathrm{~g}$. The variation of $H_{\mathrm{v}}$ with load is due to strain hardening. The rate of strain hardening is large at low loads and this effect decreases as the load is increased.

The strain hardening phenomena in polymers can be explained on the basis of spectrum of micromodes of deformation in the polymer chain (Rabinowetz and Brown 1967). Each micromode is activated by its characteristic temperature and stress conditions. When sufficient number of micromodes become active, largescale plastic deformation begins. On applying load, the polymeric specimen is subjected to strain hardening and as the load is increased, the specimen is subjected to greater strain hardening and hence $H_{\mathrm{v}}$ increases. The final saturation value of $H_{\mathrm{v}}$ observed without illumination may be due to permanent deformation caused by chain-chain slipping in polycarbonate. Van der Waals forces and relatively high molecular forces between the individual macromolecules are known to contribute to the strength of polymer (Bajpai and Datt 1986).

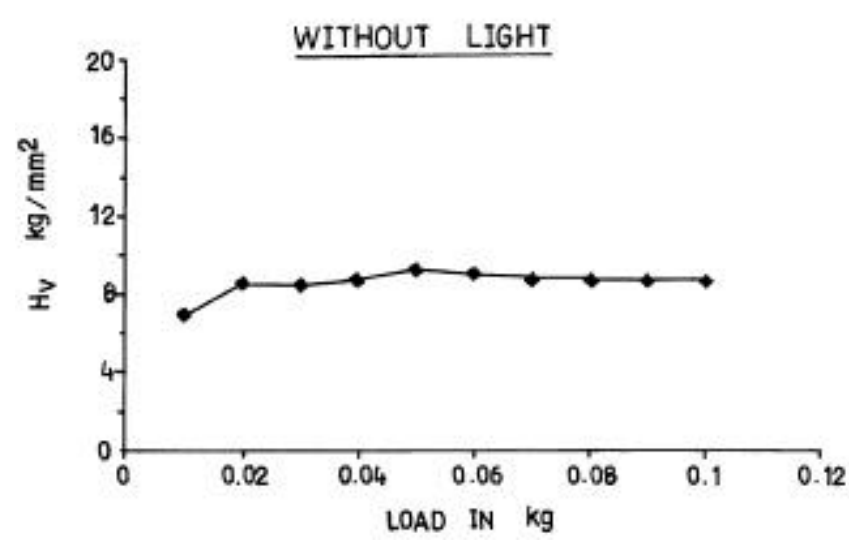

Figure 1. Microhardness of polycarbonate without illumination.

\subsection{Microhardness with illumination}

Figure 2(a) represents the variation of $H_{\mathrm{v}}$ with load for polycarbonate specimens under illumination for different exposure times of 10,20 and $30 \mathrm{~s}$ at the intensity of 27.7 arb unit, when specimens were placed at a distance of $6 \mathrm{~cm}$ from the source.

For the exposure time of $10 \mathrm{~s}$, it is observed that initially the microhardness increases with load up to $20 \mathrm{~g}$, beyond which it tends to decrease gradually up to $50 \mathrm{~g}$, thereafter, microhardness decreases rapidly and acquires
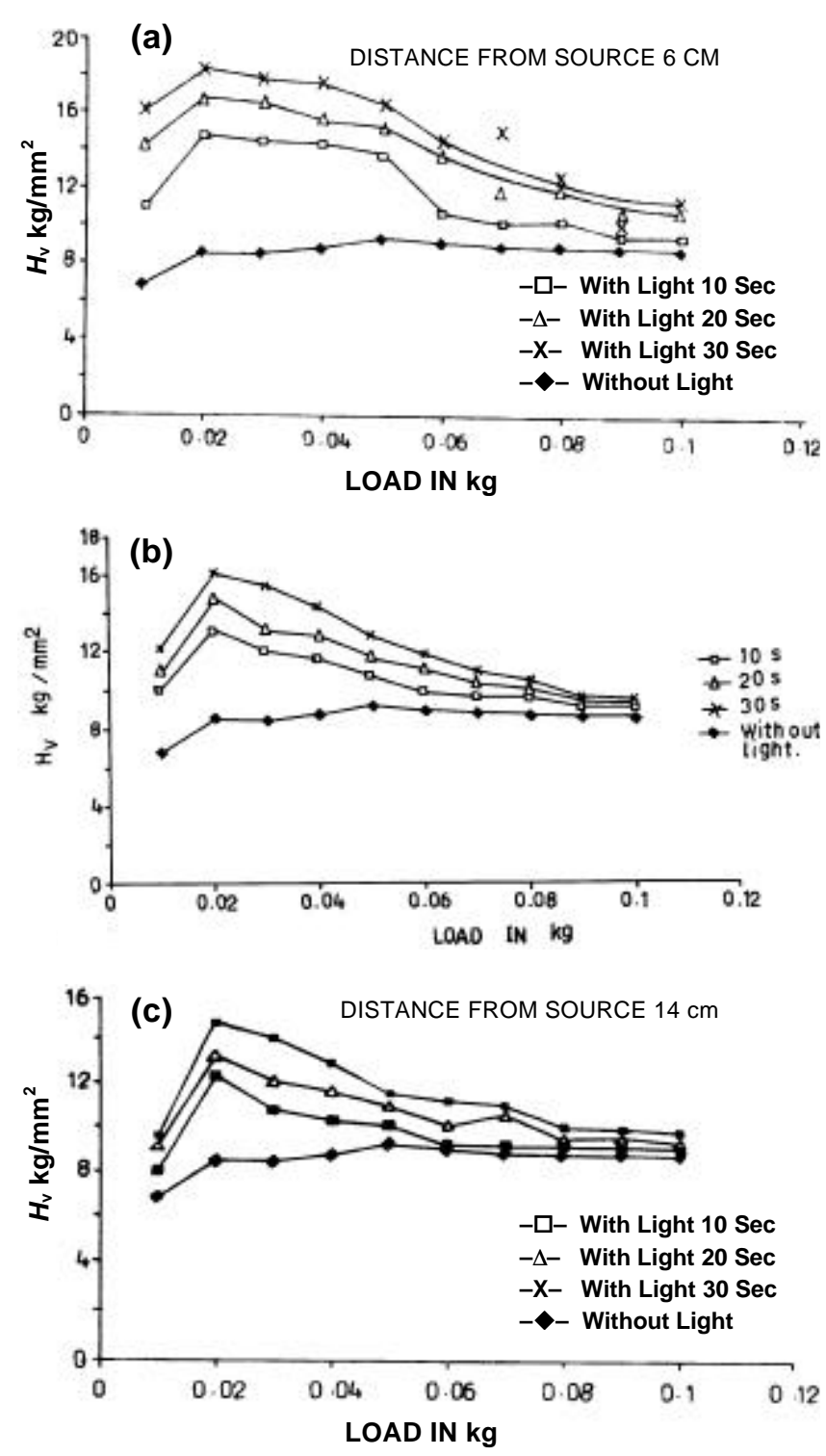

Figure 2. (a) $H_{\mathrm{v}}$ vs load curves of polycarbonate at different exposure times at fixed intensity, 27.7 arb unit (distance from source, $6 \mathrm{~cm}$ ), (b) $H_{\mathrm{v}}$ vs load curves of polycarbonate at different exposure times at fixed intensity, 10 arb unit (distance from source, $10 \mathrm{~cm}$ ) and (c) $H_{\mathrm{v}}$ vs load curves of polycarbonate at different exposure times at fixed intensity, 5 arb unit (distance from source, $14 \mathrm{~cm}$ ). 
the saturation value of $10 \cdot 16 \mathrm{~kg} / \mathrm{mm}^{2}$ at $70 \mathrm{~g}$. The effect of light is found to increase the level of microhardness as the curve for exposure time of $10 \mathrm{~s}$ is positioned higher than that without light. This corresponds to the positive photoplastic effect.

On increasing the exposure time from 10 to $20 \mathrm{~s}$ and $30 \mathrm{~s}$, similar $H_{\mathrm{v}}$-load profile is observed as that for $10 \mathrm{~s}$ exposure time. However, for specimens exposed to 20 and $30 \mathrm{~s}$ the decrease in microhardness beyond the load of $20 \mathrm{~g}$ is gradual up to $80 \mathrm{~g}$ whereas this feature is observed only up to $50 \mathrm{~g}$ in case of specimen exposed for $10 \mathrm{~s}$. It is evident from the curves that increase in the microhardness of polycarbonate on illumination at low loads is much more than that at higher loads. The exposure of specimen for longer duration increases the microhardness, which suggests that the magnitude of photoplastic effect increases with the exposure time.

Figures 2(b) and (c) also exhibit the effect of illumination on microhardness of polycarbonate for different exposure times of 10,20 and $30 \mathrm{~s}$ at the intensities of 10 and 5 arb units (when specimens are placed at distances of 10 and $14 \mathrm{~cm}$, respectively from the source). From these curves it is observed that on illumination, microhardness increases sharply up to $20 \mathrm{~g}$ and thereafter the microhardness decreases rapidly up to the load of $80 \mathrm{~g}$, beyond which the effect of load on the microhardness gets stabilized for all the exposure times as there is no appreciable change in the value of microhardness for illuminated and non illuminated specimens.

Figures 3(a)-(c) represent the $H_{\mathrm{v}}$ distance profile at loads of 20, 40 and $80 \mathrm{~g}$ for different exposure times. These curves illustrate that at low loads, the effect of exposure time on microhardness is more pronounced even at low intensity whereas at higher loads, exposure time is effective only at higher intensity. Thus it clearly indicates the photoplastic effect as revealed from the increase in the value of microhardness on illuminating the specimens.

Figure 4(a) illustrates the $H_{\mathrm{v}}$ load variation at different intensities of 27.7, 10 and 5 arb units (when specimens are placed at different distances of 6,10 and $14 \mathrm{~cm}$ from the source) for a fixed exposure time of $10 \mathrm{~s}$. From these curves, at the intensity of 27.7 arb unit (specimens at a distance of $6 \mathrm{~cm}$ ), the microhardness increases with increasing load up to the load of $20 \mathrm{~g}$ and it is approximately constant up to $50 \mathrm{~g}$. Thereafter, $H_{\mathrm{v}}$ value suddenly decreases and acquires a saturation value at $90 \mathrm{~g}$. For intensities of 10 and 5 arb units (distance of 10 and 14 $\mathrm{cm}$ ), the microhardness increases up to $20 \mathrm{~g}$, thereafter, gradually decreases up to $60 \mathrm{~g}$ and acquires saturation value at $70 \mathrm{~g}$. On comparing these curves it is observed that microhardness increases with increasing intensity. At low intensity, saturation in the value of microhardness occurs earlier than at high intensity. Figures 4(b) and (c) exhibit the effect of intensity on the microhardness for exposure times of 20 and $30 \mathrm{~s}$. These curves reveal that at high intensity, for large exposure time, the value of microhardness is relatively very high as compared to that at low intensity.

Figures 5 (a)-(c) represent the $H_{\mathrm{v}}$ vs time of exposure for loads of 20, 40 and $80 \mathrm{~g}$ at different distances (intensities). On comparing these curves it is observed that at low loads, microhardness increases linearly with increasing intensity, however, at higher loads microhardness for high intensity initially increases and rapidly attains a constant value. At low intensity, the $H_{\mathrm{v}}$ value is approximately constant. It is also observed that at high intensity,
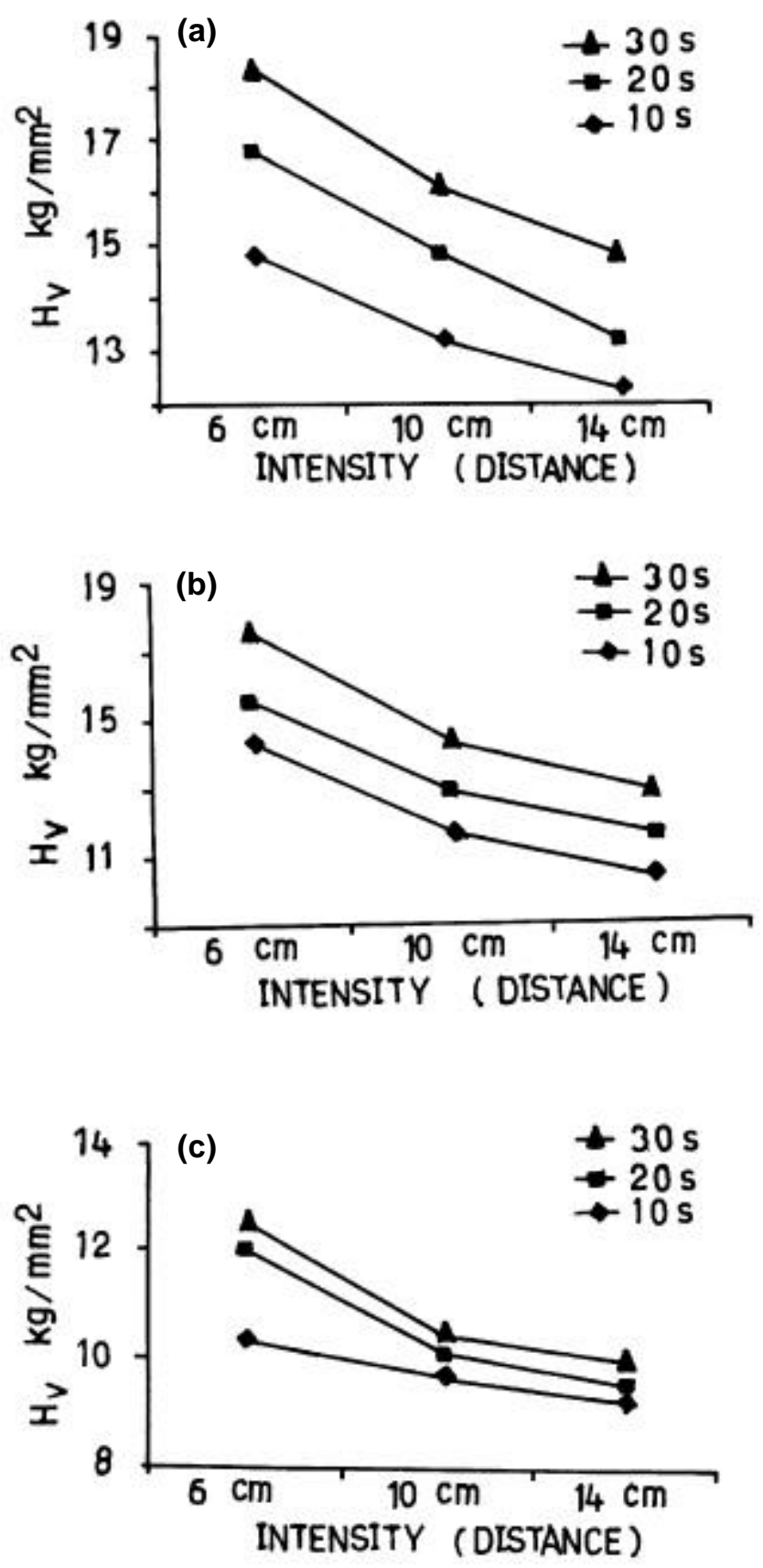

Figure 3. (a)-(c) $H_{\mathrm{v}}$ vs intensity for the loads of 20,40 and $80 \mathrm{~g}$ at different exposure times. 

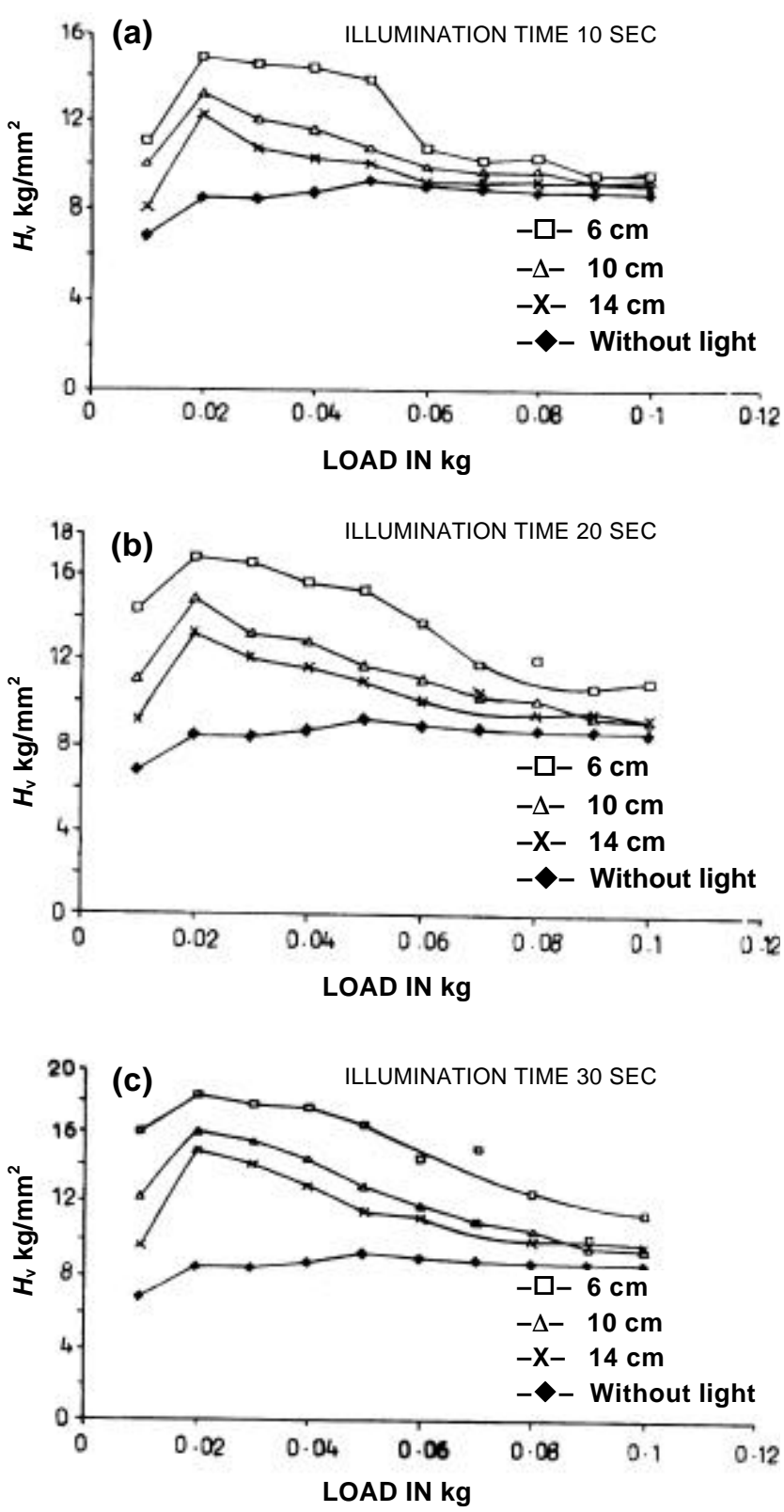

Figure 4. (a) $H_{\mathrm{v}}$ vs load at different intensities, 27.7, 10 and 5 arb units (when sample placed at different distances of 6,10 and $14 \mathrm{~cm}$ from the source) for a fixed exposure time, $10 \mathrm{~s}$, (b) $H_{\mathrm{v}}$ vs load at different intensities, 27.7, 10 and 5 arb units (when sample placed at different distances of 6,10 and $14 \mathrm{~cm}$ from the source) for a fixed exposure time, $20 \mathrm{~s}$ and (c) $H_{\mathrm{v}}$ vs load at different intensities, 27.7, 10 and 5 arb units (when sample placed at different distances of 6,10 and $14 \mathrm{~cm}$ from the source) for a fixed exposure time, $30 \mathrm{~s}$.

hardness is maximum for small value of loads and for higher loads its value decreases and becomes constant.

The increase in the exposure time at different intensities reveals that saturation in the level of microhardness occurs earlier at low intensity than at high intensity. At high intensity, for large exposure time, the value of microhardness is relatively very high as compared to that at low intensity. The higher value of $H_{\mathrm{v}}$ for the irradiated
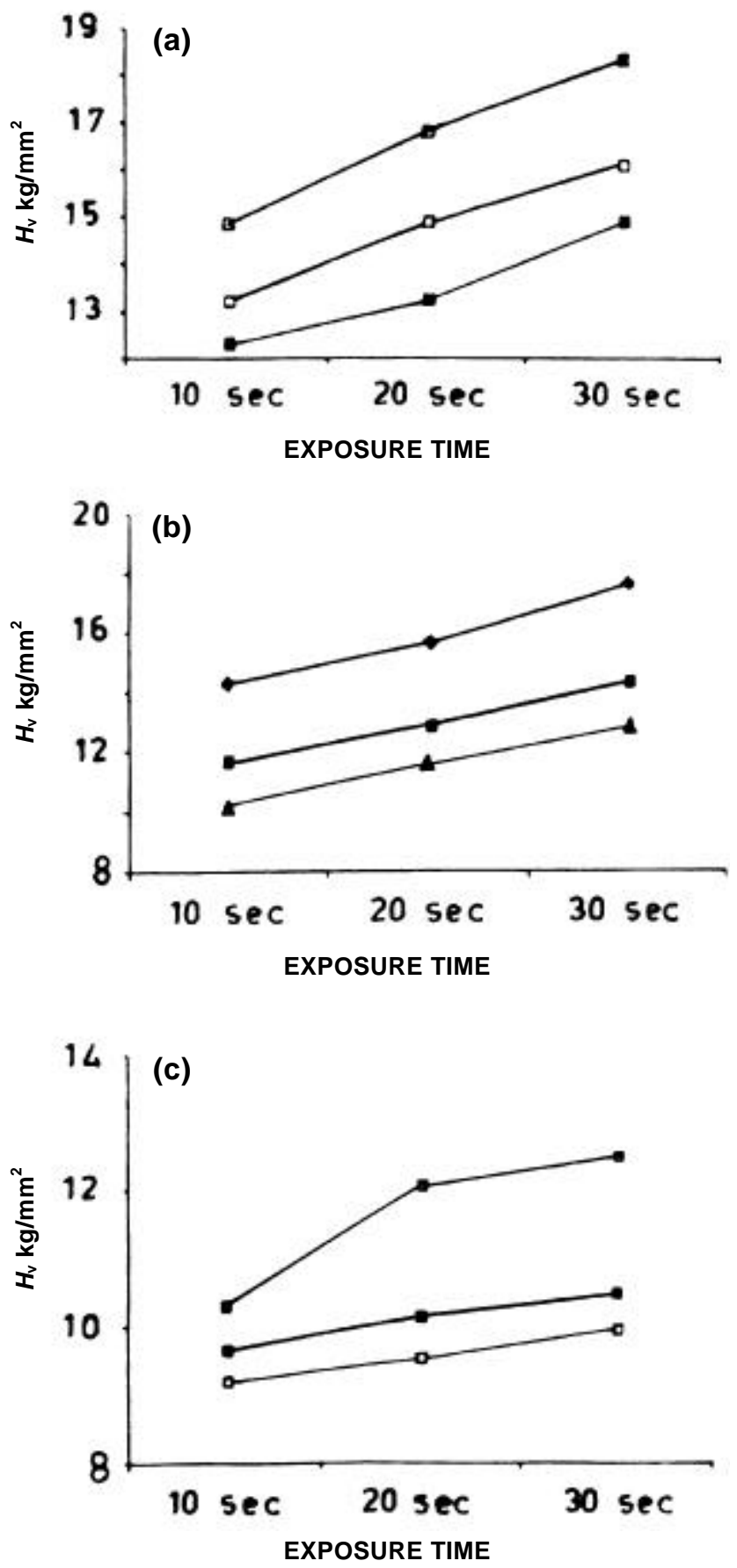

Figure 5. (a)-(c) $H_{\mathrm{v}}$ vs time of exposure for loads 20,40 and $80 \mathrm{~g}$ at different intensities, 27.7, 10 and 5 arb units (when sample placed at different distances of 6,10 and $14 \mathrm{~cm}$ from the source) for a fixed exposure time, $10 \mathrm{~s}$.

specimens as compared to the unirradiated specimens may be attributed to the radiational crosslinking in polycarbonate.

Irradiation produces various degrees of crosslinking and scissioning in the polycarbonate and thus exhibiting both increase and decrease in the deformation levels. Moreover, the crosslinking and scissioning occurs simultaneously, hence their relative ratio decides the deforma- 
tion characteristics. Irradiation imparts scissioning leading to softening of specimens. In this case crosslinking predominates and induces hardening in polycarbonate. The value of $H_{\mathrm{v}}$ for illuminated specimens when compared with those of corresponding non illuminated ones are found to have increased and this leads to positive photoplastic effect.

\section{Conclusions}

Microhardness of polycarbonate on illumination is maximum for small value of loads and for high loads it decreases and finally becomes constant. At high intensity, exposure time is more effective than at low intensity. The hardening of specimens under illumination confirms the positive photoplastic effect that causes illumination-indu- ced increase of crosslinking in polycarbonate. For high applied loads the positive photoplastic effect decreases.

\section{References}

Bajpai R and Datt S C 1986 Indian J. Pure \& Appl. Phys. 24 254

Brill W A 1966 Basic studies in photoplasticity, Ph.D. Dissertation, Stanford University, Stanford

Gonzalez A, Martin G J, de Saja J A and Rodriguez R 1986 J. Appl. Polym. Sci. 31717

Ito K 1962 Exp. Mech. 2979

Lopez J 1993 Polym. Test 12437

Mills N J 1976 J. Mater. Sci. GB 11363

Rabinowetz S and Brown N 1967 J. Polym. Sci. 5143

Zurimendi J A, Biddlestone F, Hay J N and Haward R N 1982 J. Mater. Sci. 17199 